\title{
Ipsilateral proximal and shaft femoral fractures treated with bridge-link type combined fixation system
}

\author{
Liangqi Kang ${ }^{1}$, Hui Liu', Zhenqi Ding ${ }^{1}$, Yiqiang Ding ${ }^{2}$, Wei $\mathrm{Hu}^{2}$ and Jin $\mathrm{Wu}^{1 *}(\mathbb{D}$
}

\begin{abstract}
Background: Although many treatments for ipsilateral proximal and shaft femoral fractures have been developed, controversy exists regarding their optimal management. The purpose of this retrospective study was to discuss the effectiveness of the bridge-link type combined fixation system (BCFS) and evaluate functional outcomes in treating patients with these complex fractures.
\end{abstract}

Patients and methods: We retrospectively reviewed 14 cases of ipsilateral proximal and shaft femoral fractures treated from January 2012 to December 2016. All cases were treated by BCFS combined with minimally invasive percutaneous plate osteosynthesis (MIPPO). Clinical and radiographic data were collected during regular post-operative follow-up visits. Functional outcomes were determined according to the Friedman and Wyman scoring system.

Results: The proximal femoral fractures were emergency diagnoses in 11 cases and delayed diagnoses in 3 cases. The delay time was 5-6 days, with an average of 5.3 days. The mean operation time was $179.6 \mathrm{~min}$ (range 135-231 min) with a blood loss volume that ranged from 430 to $535 \mathrm{ml}$ (average $483.6 \mathrm{ml}$ ). Follow-up was conducted in 13 cases between 9 and 30 months post-operation, with an average follow-up time of 17.3 months. The proximal femoral fractures were united in 12 cases at the final follow-up. One case had nonunion 13 months after the operation, underwent valgus intertrochanteric osteotomy, and healed 6 months later. The femoral shaft fractures obtained rigid union at the latest follow-up in 12 cases. One case endured nonunion 12 months after the operation. After the revision surgery and iliac bone grafting, the fracture healed 6 months later. Eight of the cases had good functional results, 4 had fair results, and results were poor in 1 case at the final follow-up.

Conclusions: The treatment of ipsilateral proximal femoral and shaft fractures with BCFS in combination with MIPPO demonstrated a high likelihood of union for both fractures and good functional results.

Keywords: Femoral shaft fractures, Femoral proximal fractures, Bridge-link type combined fixation system (BCFS), MIPPO

\section{Introduction}

The first case report of an ipsilateral proximal and shaft femoral fracture was published in 1953 [1]. Since then, orthopedists have paid more attention to this combined injury pattern and provided recommendations regarding

\footnotetext{
* Correspondence: wujin1983@xmu.edu.cn

'Department of Orthopedics, The Affiliated Southeast Hospital of Xiamen University, Zhangzhou 363000, China

Full list of author information is available at the end of the article
}

its diagnosis and treatment. Ipsilateral proximal and shaft femoral fracture presentation is rare, accounting for approximately $2.5-6 \%$ of femoral fractures [2]. The average patient age is 35 years, and $75 \%$ of patients are male [3]. Most cases are caused by high-impact injuries, such as motor vehicle accidents or falls, and are often associated with multisystem (head, chest, or abdominal) associated injuries [3, 4]. Twenty to $40 \%$ of patients also have ipsilateral knee injuries, including ligamentous 
injury, tibial plateau fracture, patellar fracture, or knee dislocation [4]. With the increase in high-energy injuries in recent years, the ipsilateral proximal and shaft femoral fracture morbidity rate is rising.

Attributed injury mechanisms include an axial force transmitted proximally on a flexed femur toward the acetabular roof, causing the combination of femoral shaft fracture and ipsilateral proximal femoral fracture [2-5]. Often, diagnosis of proximal femoral fracture is delayed in $19-31 \%$ of patients because of the high Injury Severity Score (ISS), other injuries associated with shaft femoral fracture, and unapparent clinical signs of the hip [6]. Surgical stabilization with implants for ipsilateral proximal and shaft femoral fractures is recommended by most published reports, and various techniques and implants have been developed to manage such fractures [2, 3, 5-9]. However, optimal treatment of these complex fractures is still debated, with different techniques and implants each having their own advantages and disadvantages. Further, the incidence of femoral fracture nonunion is $2-10 \%$, and the rate of femoral neck postoperative complications could be as high as $25 \%[2,4,10-12]$.

Based on the principles of biological osteosynthesis which emphasize both the stability of the fracture region and maintenance of soft tissue vitality, a clamp rod internal fixator (CRIF) system was designed by the AO Development Institute [13]. However, due to the lack of locking structure between plate and screws, there is poor bone-holding power; thus, CRIF is not the preferred implant for osteosynthesis of long bones [14, 15]. BCFS (Walkman Biomaterial Co., Ltd., Tianjin, China, patent number: ZL 20052 0022267.7) is a novel clamp rod internal fixation device that has been widely used in the fixation of fractures of the upper and lower limbs or pelvis. Biomechanical and clinical analysis showed favorable results with BCFS for long bone fractures [16]. To our knowledge, there are few reports regarding the treatment of ipsilateral proximal and shaft femoral fractures with $\mathrm{BCFS}$, and this retrospective study was performed to evaluate $\mathrm{BCFS}$ for the treatment of ipsilateral proximal and shaft femoral fractures.

\section{Patients and methods}

\section{Clinical data}

This study was a retrospective analysis of existing clinical cases and approved by the institutional review board. Fourteen patients (10 males and 4 females) were enrolled in the study between January 2012 and December 2016. Detailed clinical patient parameters are shown in Table 1. The average age at enrolment was 35.4 years (range: 17-60 years). Twelve patients suffered from closed fractures, and 2 had open fractures (all Gustilo I). The causes of injury included traffic accidents (10 patients) and falls from a significant height (4 patients). Two patients had fractures associated with hematopneumothorax, one with ipsilateral tibial fracture, one with liver contusion, one with traumatic brain injury, one with contralateral tibial fracture, and one with ipsilateral patella fracture. There were twelve neck fractures, one trochanteric, and one subtrochanteric fractures. According to the AO-OTA classification, there were three type A, three type $B$, and eight type $C$ femoral shaft fractures. According to Garden's classification, two femoral neck fractures were type I, seven were type II, two were type III, and one was type IV. Garden type I and II fractures are considered nondisplaced, and Garden types III and

Table 1 Clinical parameters of the patients

\begin{tabular}{|c|c|c|c|c|c|}
\hline No & Gender/age (years) & Injury mechanism & $\begin{array}{l}\text { Femoral shaft fracture } \\
\text { (Gustilo/AO-OTA type) }\end{array}$ & $\begin{array}{l}\text { Proximal femoral fracture } \\
\text { (fracture type/anatomical location) }\end{array}$ & Complicated injury \\
\hline 1 & Male/29 & Traffic accident & Closed/C & Garden I/Basicervical fracture & - \\
\hline 2 & Male/42 & Traffic accident & Open I/C & Garden II/Basicervical fracture & - \\
\hline 3 & Male/27 & Fall from height & Closed/A & Evans-Jensen II/Trochanteric fracture & Hematopneumothorax \\
\hline 4 & Male/36 & Traffic accident & Closed/C & Garden II/Basicervical fracture & - \\
\hline 5 & Male/33 & Traffic accident & Closed/B & Garden III/Basicervical fracture & Ipsilateral tibial fracture \\
\hline 6 & Male/22 & Fall from height & Closed/C & Garden II/Transcervical fracture & Liver contusion \\
\hline 7 & Male/40 & Traffic accident & Closed/A & Garden II/Basicervical fracture & - \\
\hline 8 & Female/45 & Fall from height & Closed/C & Garden IV/Basicervical fracture & Traumatic brain injury \\
\hline 9 & Male/30 & Traffic accident & Closed/C & Garden II/Basicervical fracture & - \\
\hline 10 & Female/36 & Traffic accident & Closed/C & Garden II/Basicervical fracture & Ipsilateral patella fracture \\
\hline 11 & Male/30 & Traffic accident & Closed/C & Garden III/Subcapital fracture & Contralateral tibial fracture \\
\hline 12 & Male/60 & Traffic accident & Open I/A & Seinsheimer II/Subtrochanteric fracture & - \\
\hline 13 & Female/48 & Traffic acciden & Closed/B & Garden I/Basicervical fracture & Hematopneumothorax \\
\hline 14 & Female/17 & Fall from height & Closed/B & Garden II/Basicervical fracture & - \\
\hline
\end{tabular}


IV are considered displaced [17]. Anatomically, one of the neck fractures was subcapital fracture, ten were basicervical fractures, and one was transcervical fractures. According to Evans-Jensen and Seinsheimer classification, there was one type II trochanteric fracture and one type II subtrochanteric fracture, respectively. All patients were diagnosed by clinical symptomatology, X-ray, and computer tomography (CT).

\section{Pre-operative preparation}

Proximal tibial skeletal traction was performed immediately after patients with closed fractures were admitted to the hospital (calcaneal traction was performed in patients accompanied with patella or tibial fractures). Patients with Gustilo I open fractures first underwent debridement and suturing, after which they received proximal tibial skeletal traction. All patients with open fractures received intravenous antibiotics for $24 \mathrm{~h}$ postoperatively. X-ray and CT examinations were used to visualize fracture displacement for the operative plan. As soon as their condition was stabilized, all patients underwent surgical treatment.

\section{Surgical procedure}

The patient was placed in the supine position and was under either general or epidural anesthesia. For femoral neck fractures, temporary fixation was performed with Kirschner wires after the fractures were reduced with appropriate abduction and internal rotation. For femoral trochanteric or subtrochanteric fractures, fixation was performed with a reconstruction locking plate or cortical screws after satisfactory reduction. Then, a proximal femoral anatomical clamp was placed below the apex of the greater trochanter, and, after insertion of the guide pins, 3 proximal cannulated screws were placed into the head. For femoral shaft fractures, limited open reduction was performed. After elevating the vastus lateralis muscle from the intermuscular septum, the lateral cortex of the femoral shaft was visualized. The affected limb was pulled longitudinally, and a periosteum elevator was inserted between the fracture site, manipulated to elevate the fragments. One 6-8 hole reconstruction locking plate was placed at the anterior site of the femur, and the distal and proximal ends of the fracture were each fixed with 3 unicortical locking screws. Finally, the lateral femoral condyle was exposed through a limited open approach. A distal femoral anatomical clamp was then placed on the lateral femoral condyle, and the length of the connection rods used between the proximal and distal femoral anatomical clamps was measured. Two connection rods with appropriate length were taken, three or four sliding clamps were installed on the connection rods, and then the connection rods were inserted from the distal femur into the proximal femur through the soft tissue tunnel. The distal and proximal ends of the connection rods were put into the anatomical clamps, and the position of each sliding clamp was adjusted. At last, the set screws were screwed into the clamps to tighten the connection rod and hold the clamp tightly. Reduction and femoral alignment was confirmed by fluoroscopy. Finally, the wound was irrigated and closed, and a drain was placed.

\section{Postoperative management}

The postoperative ambulatory program consisted of non-weight-bearing activities for 8-12 weeks and then gradual partial weight-bearing activities. Full-weight bearing on the affected limb was commenced once the presence of radiological consolidation was noted.

\section{Outcome assessment}

Outcome assessment after surgery was evaluated according to Friedman and Wyman scoring system [18]. A "good" result meant no limitation of activities of daily living, no pain, and loss of less than $20 \%$ of hip or knee motion. A "fair" result indicated mild limitation of activities of daily living, mild to moderate pain, and a loss of motion of $20-50 \%$. A "poor" result indicated moderate limitation of activities of daily living, severe pain, and loss of motion of more than $50 \%$. Radiological parameters included X-ray and/or CT scans were taken every 1 month post-operation to evaluate the bony fusion until they showed solid continuous callus formation. Based on previous studies [9], femoral neck fractures were considered united if anteroposterior (AP) and lateral radiographs showed that three of four cortices had trabeculae bridging the fracture site. When the patient could tolerate full-weight bearing without pain or radiological consolidation of the fracture was visible, the femoral shaft fracture was considered healed. If complete radiological consolidation of the fracture was not present by 6 months postoperatively, it was classified as a delayed union. When consolidation was not present by 12 months, the fracture was classified as nonunion [19].

\section{Results}

The mean operation time was 179.6 min (range 135-231 min) with a blood loss volume ranged from 430 to 535 $\mathrm{ml}$ (average $483.6 \mathrm{ml}$ ). Follow-up was conducted in thirteen patients between 9 and 30 months post-operation, with an average follow-up time of 17.3 months. One patient lost connection at 3-month follow-up. The proximal femoral fractures were united in 12 cases at the final follow-up. The duration for bone union ranged from 3 to 6 months, with an average of 4.2 months. One case had nonunion 13 months after the operation and underwent valgus intertrochanteric osteotomy and healed 6 months later. The femoral shaft fractures 
obtained rigid union in 12 cases at the latest follow-up. The duration for bone union ranged from 3 to 7 months, with an average of 5.0 months. One case endured nonunion 12 months after the operation. After the revision surgery and iliac bone grafting, the fracture healed 6 months later. According to Friedman and Wyman scoring system, 8 of the cases had a good functional result, in 4 the result was fair, and in 1, it was poor at the final follow-up. The complications included infection in 1, varus angulation of femoral neck in 1 , nonunion of femoral neck fracture in 1 , nonunion of femoral shaft fracture in 1 , and avascular necrosis of femoral head in 1 (Table 2). Typical cases are shown in Fig. 1 (patient 3) and Fig. 2 (patient 12).

\section{Discussion}

Although ipsilateral proximal and shaft femoral fractures are not common, they present a diagnostic and therapeutic challenge for orthopedic surgeons. The majority of the patients in the reported case series were young males with high-energy trauma. Due to the frequency with which the injury is seen in vehicle crash front seat drivers and passengers, the typical damage mechanism has been postulated to be the result of a longitudinal compression force on a flexed and abducted hip. The femoral shaft fracture is typically comminuted, located in the middle third of the diaphysis, and open in 15$33 \%$ of cases. The femoral neck fracture is usually basicervical, vertically oriented, and nondisplaced in $60 \%$ of cases [20].
Diagnosis of proximal femoral fracture is easily delayed or missed. The incidence of delayed diagnosis is around $19-31 \%$, and the incidence of missed diagnosis may be $13-31 \%$ [6]. Early diagnosis is generally difficult because the fracture is most often basilar and the symptoms are mild in the early phase of the disease. Furthermore, surgeons pay more attention to the often lifethreatening associated injuries (head, chest, or abdominal) [4]. Therefore, patients with femoral shaft fractures should undergo thorough radiography of the pelvis and both hips as a standard protocol. Additionally, CT scan examination should be performed if necessary. However, a nondedicated CT scan does not always reveal the femoral neck fracture preoperatively [21]. Objective sound waves can also be used as a diagnostic test or screening tool in the assessment of occult hip fractures [22]. Moreover, a recent study found that rapid limited-sequence pelvis MRI for patients with femoral shaft fractures could identify femoral neck fractures that were not diagnosed on thin-cut high-resolution CT [23]. In our present series, nine of the patients were young males (64.2\%), two had open femoral shaft fractures (14.3\%), seven had multisystem injuries (50.0\%), and two had ipsilateral knee injuries (14.3\%). Further, nine of the neck fractures were nondisplaced $(75.0 \%)$, one was a subcapital fracture $(8.3 \%)$, ten were basicervical fractures $(83.3 \%)$, and one was a transcervical fracture (8.3\%). Delayed diagnosis was observed in 3 cases (21.4\%). The average delay time was $5.3 \mathrm{~d}$ (range, 5-6 days), and all of these cases were transferred from other departments to orthopedics. All of

Table 2 Clinical parameters of the patients

\begin{tabular}{|c|c|c|c|c|c|c|c|c|c|}
\hline No & $\begin{array}{l}\text { Delayed } \\
\text { diagnosis } \\
\text { (days) }\end{array}$ & $\begin{array}{l}\text { Time to surgery } \\
\text { (days) }\end{array}$ & $\begin{array}{l}\text { Operation time } \\
\text { (mins) }\end{array}$ & Blood loss (ml) & $\begin{array}{l}\text { Follow-up } \\
\text { (months) }\end{array}$ & $\begin{array}{l}\text { Neck union } \\
\text { (months) }\end{array}$ & $\begin{array}{l}\text { Shaft union } \\
\text { (months) }\end{array}$ & Outcomes & Complications \\
\hline 1 & - & 3 & 148 & 460 & 18 & 3 & 5 & Good & - \\
\hline 2 & 5 & 11 & 162 & 510 & 22 & 4 & 4 & Good & Superficial infection \\
\hline 3 & - & 7 & 206 & 565 & 30 & 6 & 6 & Good & - \\
\hline 4 & - & 4 & 155 & 455 & 17 & 4 & 17 & Fair & Femoral shaft nonunion \\
\hline 5 & - & 8 & 197 & 525 & 12 & 5 & 4 & Fair & $\begin{array}{l}\text { Femoral neck varus } \\
\text { angulation }\end{array}$ \\
\hline 6 & 6 & 14 & 183 & 500 & 25 & 4 & 5 & Good & - \\
\hline 7 & - & 2 & 174 & 450 & 9 & 3 & 3 & Good & - \\
\hline 8 & - & 11 & 191 & 495 & 21 & 5 & 6 & Good & - \\
\hline 9 & - & 3 & 178 & 445 & 14 & 4 & 5 & Good & - \\
\hline 10 & - & 3 & 194 & 510 & 12 & 3 & 7 & Fair & - \\
\hline 11 & - & 6 & 231 & 535 & 16 & 5 & 6 & Poor & $\begin{array}{l}\text { Osteonecrosis of the } \\
\text { femoral }\end{array}$ \\
\hline 12 & - & 20 & 166 & 520 & Lost & - & - & - & head \\
\hline 13 & 5 & 9 & 157 & 430 & 19 & 19 & 4 & Fair & - \\
\hline 14 & - & 3 & 135 & 455 & 11 & 4 & 5 & Good & Femoral neck nonunion \\
\hline
\end{tabular}



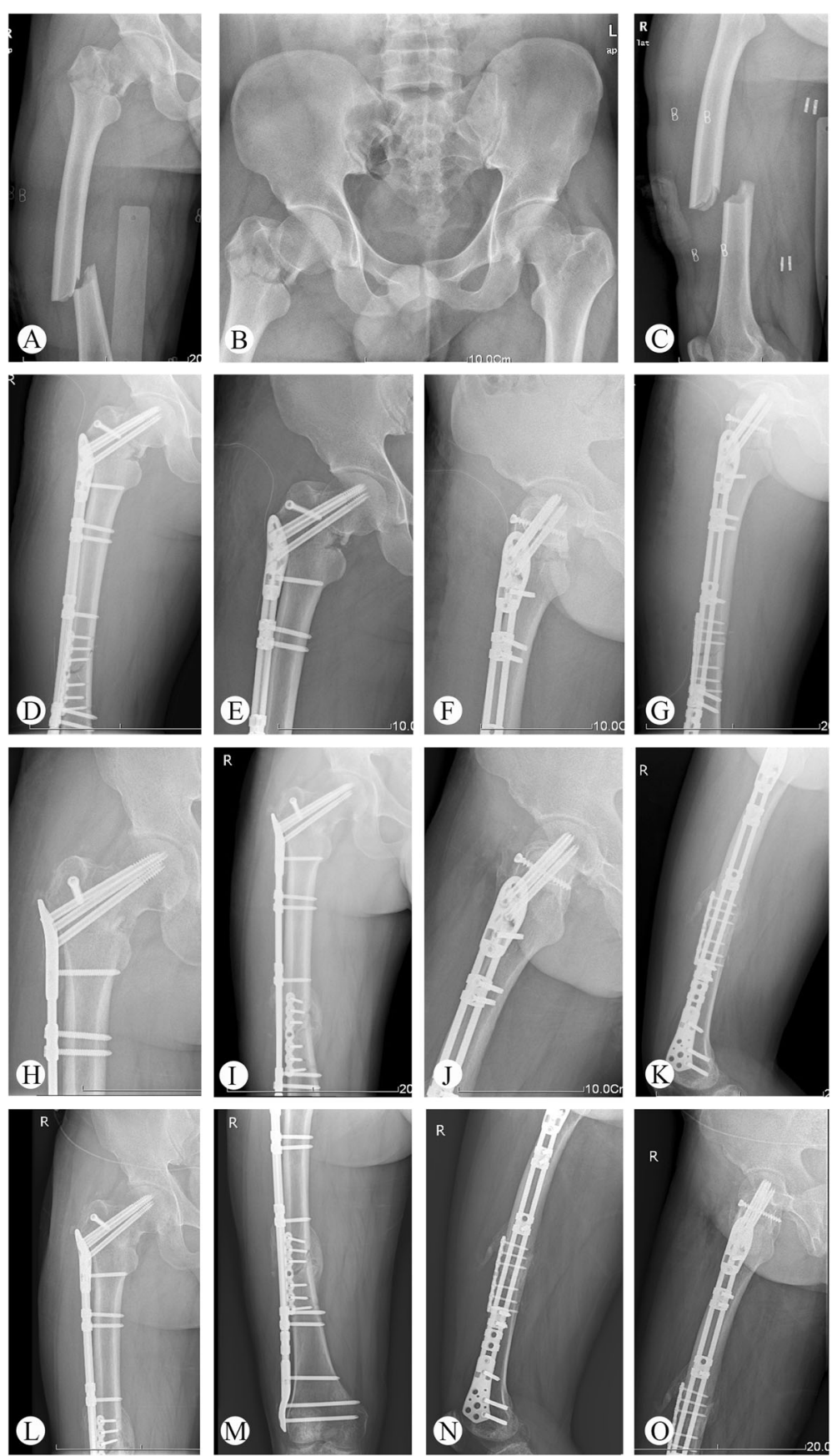

Fig. 1 Representative images of patient 3. a-c X-ray at admission. $\mathbf{d}-\mathbf{g}$ X-ray at 7 days after the operation. $\mathbf{h}-\mathbf{k}$ X-ray at 4 months follow-up. I-o X-ray at last follow-up

the results in our study were consistent with previous reports.

Non-operative treatment of either the proximal femoral fracture or shaft fracture is generally avoided except in extenuating circumstances, and poor results have been reported [24]. A series of surgical treatment strategies have been described for this combined injury. Orthopedic surgeons need to consider three issues for an optimal preoperative plan: (1) appropriate timing of surgical fixation, (2) which fracture to stabilize first, and (3) the optimal internal fixation for these complex fractures. The purpose of ipsilateral proximal and shaft femoral fracture surgical management is to achieve the anatomical reduction of the proximal femoral fracture and rigid fixation of both fractures. Studies have recommended that the operation be performed 

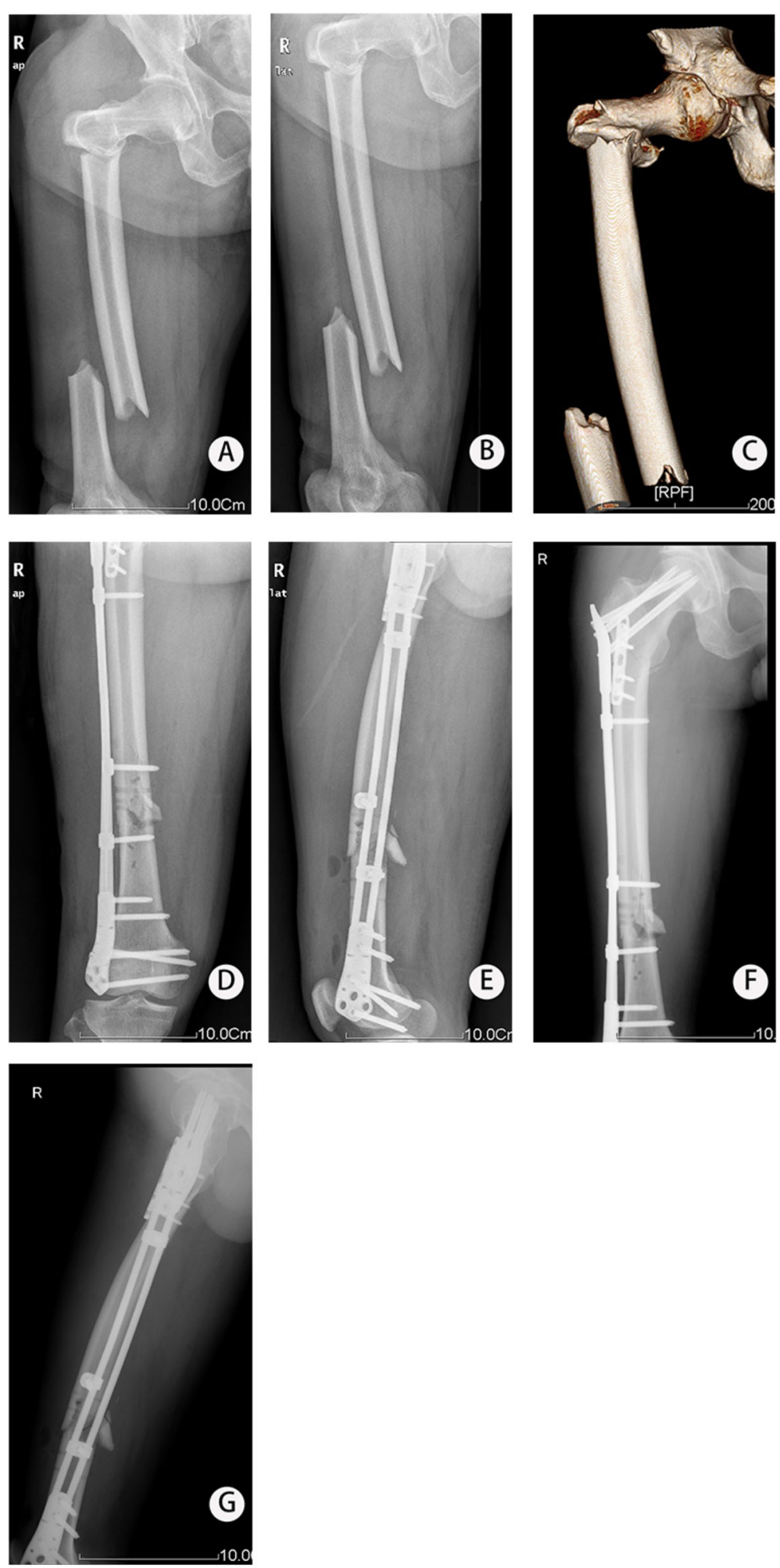

Fig. 2 Representative images of patient 12 who lost connection at 3-month follow-up. a-c X-ray at admission. $\mathbf{d}$-i X-ray at 3 days after operation 
within $8 \mathrm{~h}, 12 \mathrm{~h}, 24 \mathrm{~h}, 72 \mathrm{~h}$, or 1 week [9, 25-27]. It is generally accepted that early surgical treatment can reduce patient complications and morbidity and allow for earlier functional exercise and rehabilitation. Therefore, after the patient's general condition has stabilized, early reduction and fixation of this combined injury pattern are necessary. There is no consensus as to which fracture should be managed first. Some recommend that the femoral neck fracture should be always provisionally fixed first, either by closed or open methods, to avoid displacement of a nondisplaced or minimally displaced fracture and to ensure anatomic reduction and optimal stabilization of the neck, preventing osteonecrosis and nonunion [8]. Others advocate for fixation of the femoral shaft first to allow better control of the leg during the more technically challenging femoral neck reduction [28]. Generally speaking, we favored that the fixation should depend on the femoral neck fracture pattern. This therapeutic strategy is satisfactory with nondisplaced neck fractures, as neck fractures could be fixed in situ and further displacement is prevented. Otherwise, the femoral shaft should be fixed first to allow for better control of the leg during the reduction of the displaced femoral neck fracture.

Various constructs and surgical techniques have been described for the treatment of ipsilateral proximal and shaft femoral fractures, including single constructs or dual constructs for both fractures $[4,6,7,25,29,30]$. Single constructs include reconstruction intramedullary nails with interlocked screw fixation for the neck fracture via the nail, long proximal femoral nails (PFN-long) with anti-rotation, long sliding hip screws, and long proximal femoral locking plates $[7,9,20]$. Some favor the reconstruction nail, which allows possible closed reduction and biomechanical fixation with minimal incision, achieving biological fixation of both fractures and reduced intraoperative blood loss. However, this advantage may be outweighed by the high technical demand of accurately placing the proximal screws into the head and neck [7]. Additionally, reconstruction nailing should not be performed in displaced femoral neck fractures because it is difficult to achieve reduction [7]. PFN-long could stabilize and fix both fractures simultaneously, as described by Wang et al. [9]. Biomechanical studies have also shown that the helical blade could provide higher stability than the lag screw [31]. However, the availability of only three nail lengths and one diameter presents certain drawbacks [9].

Dual constructs include (1) retrograde nailing combined with dynamic hip screw (DHS) or lag screws [6]; (2) a dynamic compression plate (DCP) with DHS, cannulated cancellous screws, or a proximal femoral locking plate [2]; and (3) antegrade femoral intramedullary nails with cannulated cancellous screws [27]. When compared with single constructs, dual constructs have many advantages, such as easier immobilization and superior biomechanical fixation [7, 8]. However, their clinical application is limited by complications related to the knee, risk of fracture nonunion (particularly for retrograde nailing combined with DHS or lag screws), greater intraoperative trauma, and higher implant cost [28].

The rate of avascular necrosis of the femoral head in ipsilateral proximal and shaft femoral fractures is lower than that seen with isolated femoral neck fractures. In these fractures, the reported incidence in various series has ranged from 1.2 to 5\% [5]. Nonunion of the femoral neck can also occur. In a series of 95 patients treated with femoral neck screw fixation and retrograde reamed intramedullary nailing, the nonunion rate of the femoral neck was only $2 \%$ [32]. Complications related to ipsilateral femoral shaft fractures are more common than for the neck in almost all published series. Compared to isolated femoral shaft fractures, associated shaft fractures have a higher average time to the union (20.3-27.3 weeks) and higher rates of nonunion $0-23 \%$ and malunion $3.7-40 \%$ [33, 34]. This may be due to a difference in treatment, an inherent injury characteristic, a result of post-operative protocol changes or a combination of these factors [24]. In our present series, the rate of avascular necrosis and nonunion of the femoral head was $8.3 \%(1 / 12)$, respectively. The nonunion of femoral shaft fracture was found in one case (7.7\%). All of the results were in accord with previous reports.

In order to minimize complications and trauma, all cases in our study were treated by BCFS in combination with MIPPO. The BCFS was comprised of clamps, connection rods, screws (set screw, locking screw, and cortical screw), and other accessory instruments (Supplemental material). Based on the patient needs, the surgeon chose different sizes of clamps and connection rods during the operation. When the set screw was screwed into the clamp, its nut tightened the connection rod and held the clamp tightly. Thus, the clamps, screws, and connection rods were locked as a whole to support firm and stable fixation of both the proximal and shaft femoral fractures. Compared to other treatment methods, the BCFS has relatively easy application and less trauma. Four different treatment methods (antegrade reamed intramedullary nailing and cancellous screw, DHS and low-contact dynamic compression plate (LCDCP), cancellous screw and LCDCP, and reconstruction nailing) were used for 43 patients with ipsilateral femoral neck and shaft fractures; these procedures had a mean surgery duration of $280 \mathrm{~min}$ and resulted in a mean blood loss of $428.6 \mathrm{ml}$ [35]. The BCFS takes 179.6 min operation time with an average blood loss volume of $483.6 \mathrm{ml}$, reflecting the relative ease of manipulation. Furthermore, the curative effects, including fracture healing, complications, and postoperative 

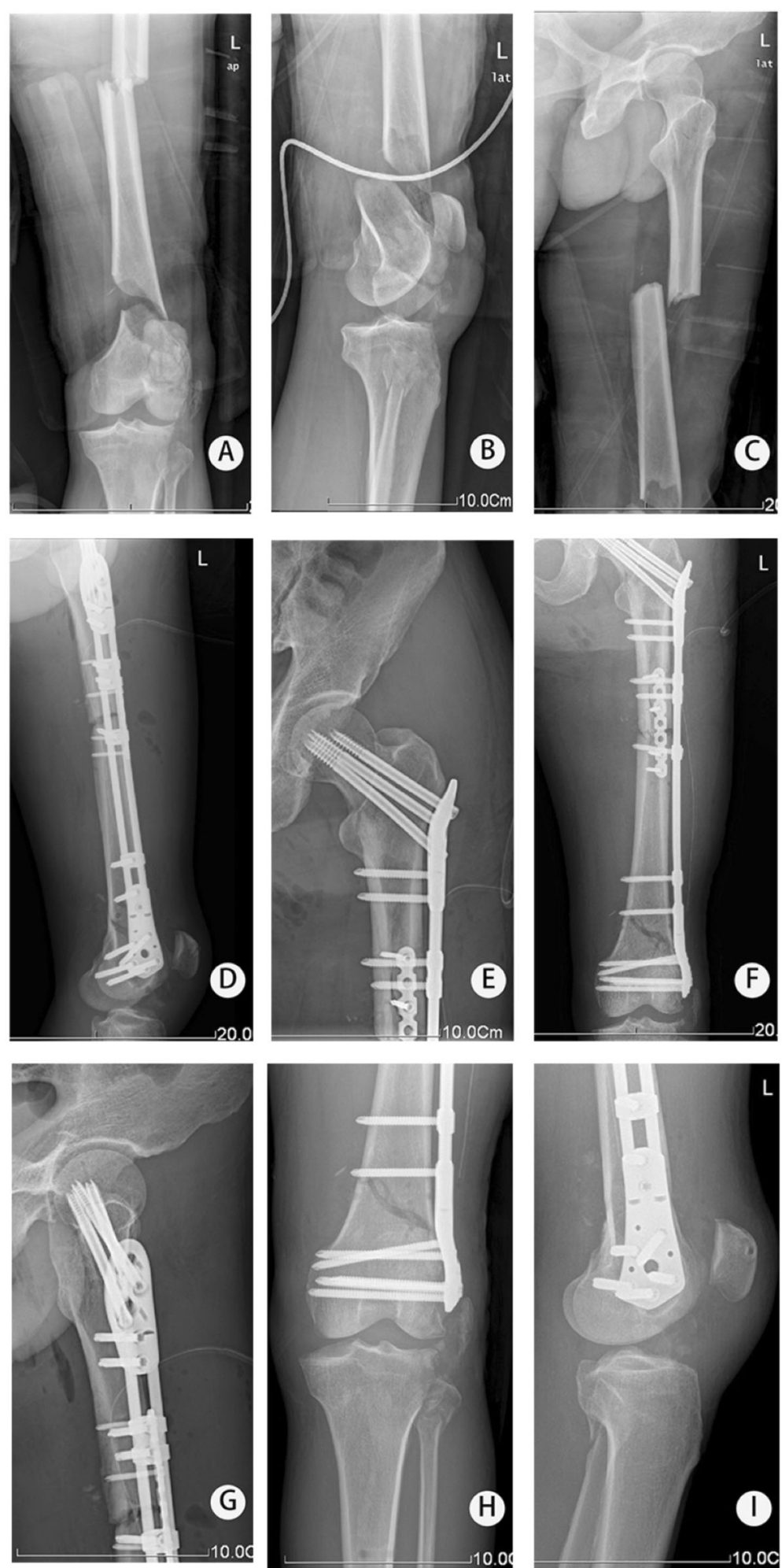

Fig. 3 A 63-year-old male who suffered a motor vehicle accident and dignosed as ipsilateral femoral neck, shaft, and distal fracture. a-c X-ray at admission. $\mathbf{d}$-i X-ray at 5 days after the operation 
functional indexes, are basically consistent with previous literature [35]. Moreover, BCFS has a wide scope of application, even in the treatment of ipsilateral fractures of the femoral neck, shaft, and distal end cases (Fig. 3).

\section{Conclusion}

Here, we have presented our results from treating concomitant ipsilateral fractures of the proximal femur and femoral shaft by BCFS. Our summarized clinical effects show that BCFS is a good option for the treatment of complex fractures with biological fixation of both fractures and satisfactory results. However, future studies featuring longer outcome measures and a higher number of patients are still needed.

\section{Supplementary information}

Supplementary information accompanies this paper at https://doi.org/10. 1186/s13018-020-01929-7.

\section{Additional file 1}

\section{Abbreviations}

AP: Anteroposterior; BCFS: Bridge-link type combined fixation system; CRIF: Clamp rod internal fixator; CT: Computer tomography; DCP: Dynamic compression plate; DHS: Dynamic hip screw; ISS: Injury Severity Score; LCDCP: Low-contact dynamic compression plate; MIPPO: Minimally invasive percutaneous plate osteosynthesis

\section{Acknowledgements}

We thank all participants in this study for their enthusiasm, tireless work, and sustained support

\section{Authors' contributions}

LK: Writing the paper, data analysis, and collection. HL: Searching the related articles and data analysis. ZD: Data collection. YD: Writing the paper, data analysis, and collection. WH: Searching the related articles and data analysis. JW: Data collection, performed surgeries, and the research team manager. All authors have contributed significantly and are in agreement with the content of the manuscript. The authors read and approved the final manuscript.

\section{Funding}

The authors disclosed receipt of the following financial support for the research, authorship, and/or publication of this article. This work was supported by grants from the Nanjing Military Region Medical Scientific and Technical Innovation Foundation Projects of People's Liberation Army of China (ZD15029).

\section{Ethics approval and consent to participate}

This study was a retrospective and clinical study and approved by the Xiamen University institutional review board. Written informed consent was obtained from all patients preoperatively.

\section{Consent for publication}

All authors are in agreement with the content of the manuscript and have approved the manuscript for submission.

\section{Competing interests}

The authors declared no potential conflicts of interest with respect to the research, authorship, and/or publication of this article.

\section{Author details}

'Department of Orthopedics, The Affiliated Southeast Hospital of Xiamen University, Zhangzhou 363000, China. ${ }^{2}$ The Affiliated Southeast Hospital of Xiamen University, Zhangzhou 363000, China.
Received: 21 May 2020 Accepted: 25 August 2020

Published online: 10 September 2020

\section{References}

1. Delaney WM, Street DM. Fracture of femoral shaft with fracture of neck of same femur; treatment with medullary nail for shaft and Knowles pins for neck. J Int Coll Surg. 1953:19:303-12.

2. Chen CM, Chiu FY, Lo WH, Chuang TY. Ipsilateral hip and distal femoral fractures. Injury. 2000;31:147-51.

3. Cannada LK, Viehe T, Cates CA, et al. A retrospective review of high-energy femoral neck-shaft fractures. J Orthop Trauma. 2009;23:254-60.

4. Alho A. Concurrent ipsilateral fractures of the hip and femoral shaft: a metaanalysis of 659 cases. Acta Orthop Scand. 1996:67:19-28.

5. Hak DJ, Mauffrey C, Hake M, Hammerberg EM, Stahel PF. Ipsilateral femoral neck and shaft fractures: current diagnostic and treatment strategies. Orthopedics. 2015;38:247-51.

6. Wolinsky PR, Johnson KD. Ipsilateral femoral neck and shaft fractures. Clin Orthop Relat Res. 1995;318:81-90.

7. Singh R, Rohilla R, Magu NK, Siwach R, Kadian V, Sangwan SS. Ipsilateral femoral neck and shaft fractures: a retrospective analysis of two treatment methods. J Orthop Traumatol. 2008;9:141-7.

8. Oh CW, Oh JK, Park BC, et al. Retrograde nailing with subsequent screw fixation for ipsilateral femoral shaft and neck fractures. Arch Orthop Trauma Surg. 2006;126:448-53.

9. Wang WY, Liu L, Wang GL, Fang Y, Yang TF. Ipsilateral basicervical femoral neck and shaft fractures treated with long proximal femoral nail antirotation or various plate combinations: comparative study. J Orthop Sci. 2010;15: 323-30

10. Okcu G, Aktuglu K. Antegrade nailing of femoral shaft fractures combined with neck or distal femur fractures. A retrospective review of 25 cases, with a followup of 36-150 months. Arch Orthop Trauma Surg. 2003;123(10):544-50.

11. Alho A. Concurrent ipsilateral fractures of the hip and shaft of the femur. A systematic review of 722 cases. Ann Chir Gynaecol. 1997:86:326-36.

12. Swiontkowski MF, Hansen ST Jr, Kellam J. Ipsilateral fractures of the femoral neck and shaft. A treatment protocol. J Bone Joint Surg Am. 1984;66(2):260-8.

13. Schwandt CS. User manual for the $4.5 / 5.5 \mathrm{~mm}$ VetFix: development of a new veterinary internal fixation system, from the prototype to the final version.. Thesis. Switzerland: University of Bern; 2001

14. Gamper S, Steiner A, Nuss K et al. Clinical evaluation of the CRIF 4.5/5.5 system for long-bone fracture repair in cattle. Vet Surg. 2006;35:361-8.

15. Zahn K, Frei R, Wunderle D, et al. Mechanical properties of 18 different $A O$ bone plates and the clamp-rod internal fixation system tested on a gap model construct. Vet Comp Orthop Traumatol. 2008;21:185-94.

16. Wang DX, Xiong $Y$, Deng $H$, et al. Biomechanical analysis and clinical effects of bridge combined fixation system for femoral fractures. Proc Inst Mech Eng H. 2014:228:899-907.

17. Gardern RS. Stability and union in subcaptial fractures of the femur. J Bone Joint Surg Br. 1964;46:630-47.

18. Friedman RJ, Wyman ET Jr. Ipsilateral hip and femoral shaft fractures. Clin Orthop Relat Res. 1986;208:188-94.

19. Krastman P, Welvaart WN, Breugem SJ, van Vugt AB. The Holland nail: a universal implant for fractures of the proximal femur and the femoral shaft. Injury. 2004;35:170-8.

20. Jones CB, Walker JB. Diagnosis and management of ipsilateral femoral neck and shaft fractures. J Am Acad Orthop Surg. 2018;26:e448-54.

21. Heiney JP, Leeson MC, Vrabec GA. Delayed diagnosis of an ipsilateral femoral neck fracture with an associated femoral shaft fracture in light of a negative computed tomography scan. J Trauma. 2009;67:E129-31.

22. Jawad Z, Odumala A, Jones M. Objective sound wave amplitude measurement generated by a tuning fork. An analysis of its use as a diagnostic tool in suspected femoral neck fractures. Injury. 2012;43:835-7.

23. Rogers NB, Hartline BE, Achor TS, et al. Improving the diagnosis of ipsilateral femoral neck and shaft fractures: a new imaging protocol. J Bone Joint Surg Am. 2020;102:309-14.

24. Boulton $\mathrm{CL}$, Pollak AN. Special topic: ipsilateral femoral neck and shaft fractures - does evidence give us the answer? Injury. 2015;46:478-83.

25. Lawson E, Madougou S, Chigblo P, et al. Ipsilateral proximal and shaft femoral fractures. Chin J Traumatol. 2017;20:155-7.

26. Chaturvedi S, Sahu SC. Ipsilateral concomitant fractures of the femoral neck and shaft. Injury. 1993;24:243-6. 
27. Parfenchuck TA, Carter LW, Young TR. Ipsilateral fractures of the femoral neck and shaft. Orthop Rev. 1993;22:356-63.

28. Bhandari M. Ipsilateral femoral neck and shaft fractures. J Orthop Trauma. 2003:17:138-40.

29. Spitler CA, Kiner D, Swafford R, Bruce J, Nowotarski P. Treatment of ipsilateral femoral neck and shaft fractures with cannulated screws and antegrade reconstruction nail. J Orthop Trauma. 2020;34:e176-80.

30. Gadegone W, Lokhande V, Salphale Y, Ramteke A. Long proximal femoral nail in ipsilateral fractures proximal femur and shaft of femur. Indian J Orthop. 2013;47:272-7.

31. Strauss E, Frank J, Lee J, Kummer FJ, Tejwani N. Helical blade versus sliding hip screw for treatment of unstable intertrochanteric hip fractures: a biomechanical evaluation. Injury. 2006;37:984-9.

32. Ostrum RF, Tornetta P 3rd, Watson JT, Christiano A, Vafek E. Ipsilateral proximal femur and shaft fractures treated with hip screws and a reamed retrograde intramedullary nail. Clin Orthop Relat Res. 2014;472:2751-8.

33. Randelli P, Landi S, Fanton F, Hoover GK, Morandi M. Treatment of ipsilateral femoral neck and shaft fractures with the Russell-Taylor reconstructive nail. Orthopedics. 1999;22:673-6.

34. Vidyadhara S, Rao SK. Cephalomedullary nails in the management of ipsilateral neck and shaft fractures of the femur--one or two femoral neck screws? Injury. 2009;40:296-303.

35. Tsai CH, Hsu HC, Fong YC, Lin CJ, Chen YH, Hsu CJ. Treatment for ipsilateral fractures of femoral neck and shaft. Injury. 2009;40:778-82.

\section{Publisher's Note}

Springer Nature remains neutral with regard to jurisdictional claims in published maps and institutional affiliations.

Ready to submit your research? Choose BMC and benefit from:

- fast, convenient online submission

- thorough peer review by experienced researchers in your field

- rapid publication on acceptance

- support for research data, including large and complex data types

- gold Open Access which fosters wider collaboration and increased citations

- maximum visibility for your research: over $100 \mathrm{M}$ website views per year

At $\mathrm{BMC}$, research is always in progress.

Learn more biomedcentral.com/submissions 